\title{
INVESTIGACIONES
}

\section{Estrategias Didácticas y Organizativas: Atender a la Diversidad en las Escuelas de Cultura y Difusión Artística}

\author{
Didactic and Organizational Strategies: \\ Attend to Diversity in the Escuelas de Cultura y Difusión Artística
}

\section{Cony Villarroel Raimilla ${ }^{a}$, José Luís Muñoz Moreno ${ }^{a}$}

${ }^{a}$ Departamento de Pedagogía Aplicada, Universidad Autónoma de Barcelona. villarroelcony@gmail.com, joseluis.munoz@uab.cat

\section{RESUMEN}

Este artículo presenta una investigación que tiene por objetivo analizar las estrategias institucionales para la atención a la diversidad que desarrollan las Escuelas de Cultura y Difusión Artística (Chile). El estudio emplea metodología de carácter mixto, mediante un estudio de caso. Para ello, se analiza el caso de la Escuela de Cultura y Difusión Artística de Puerto Montt utilizando las técnicas de análisis documental, cuestionario y entrevista. Algunas conclusiones permiten evidenciar el trabajo del centro escolar con la diversidad mediante la articulación de diferentes estrategias didácticas y organizativas para promover el aprendizaje integral de los estudiantes y facilitar procesos de mejora permanente.

Palabras clave: atención a la diversidad, inclusión educativa, estrategias institucionales, formación integral.

\begin{abstract}
This article presents a research that aims to analyze the institutional strategies for attention to diversity developed by the Escuelas de Cultura y Difusión Artística (Chile). The study uses mixed methodology, through a case study. For this, it's analyzed the case of the Escuela de Cultura y Difusión Artística of Puerto Montt using the techniques of documentary analysis, questionnaire, and interview. Some conclusions allow to demonstrate the work of the school with diversity through the articulation of different teaching and organizational strategies to promote the students' integral learning and facilitate processes of permanent improvement.
\end{abstract}

Key words: attention to diversity, educational inclusion, institutional strategies, integral development.

\footnotetext{
* El primer autor contó con el apoyo económico de la Agenda Nacional de Investigación y Desarrollo (ANID) / Programa de Beca / MAGÍSTER PROFESIONALES DE LA EDUCACIÓN-NOVENA BECAS CHILE / 2016-77170025.
} 


\section{INTRODUCCIÓN}

Nuestra sociedad actual exige cambios en la educación que les permitan garantizar su derecho a una educación equitativa y de calidad que valore y atienda a su diversidad. Bajo esta perspectiva, los centros escolares con orientación inclusiva se transforman en los medios más eficaces para dar respuesta a la diversidad (UNESCO, 2009; Payà, 2017). La reflexión en torno a las necesidades de los estudiantes y su contexto supone el principio regulador en la concreción de la respuesta educativa de los centros que permite realizar cambios y plantear estrategias a nivel organizativo y didáctico para atender a la diversidad (Gairín, 1998; Gómez, 2005; Tomlison, 2005; Álvarez, Grau y Tortosa, 2014).

A través de los años, la inclusión educativa ha tomado una direccionalidad progresista, evolucionando en sí misma y manteniendo el principio que la caracteriza: todos los niños y jóvenes, independiente de sus diferencias sociales, económicas, culturales, étnicas, de aprendizaje, entre otras, deben tener igualdad de oportunidades educativas en el sistema escolar (Echeita, 2006; Parra, 2011; UNESCO, 2009; UNESCO, 2017). En esta línea, la inclusión educativa apuesta por garantizar el derecho universal a la "Educación Para Todos" en la búsqueda por proporcionar mayor equidad y calidad de la educación, lo que debe calar tanto en las decisiones de la política educativa como en la respuesta educativa de los centros escolares para atender la diversidad (Escarbajal, Mirete, Maquilón, Izquierdo, López, Orcajada y Sánchez, 2012; Gairín, 2012).

En Chile, concretamente, se identifican diferentes políticas educativas para la atención a la diversidad, como parte del marco normativo y legal que defienden el derecho a la igualdad de oportunidades en educación, lo que supone un interés y actualización permanente hacia la inclusión educativa, tales como la Ley Indígena (Ley no 19.253 del 93), el Programa de Educación Intercultural Bilingüe (EIB), creado por el MINEDUC y la Corporación Nacional de Desarrollo Indígena (CONADI), políticas de acceso a la educación para los inmigrantes o el Programa de Integración Escolar (PIE) para la atención de estudiantes con N.E.E. Así como también, la actual Ley de Inclusión Escolar, promulgada en el año 2015.

Por otra parte, surgen otras iniciativas que permiten reconocer la preocupación del país por mejorar la calidad y equidad en la educación. Destaca la creación de las Escuelas de Cultura y Difusión Artística que ofrecen a todos sus estudiantes la enseñanza de un modelo educativo diferente basado en el desarrollo del arte y la cultura, en donde predomina la visión de la persona y la diversidad de sus experiencias y valores.

Las Escuelas de Cultura y Difusión Artística de Chile son instituciones educativas de dependencia municipal, particular subvencionado, particular pagado o de administración delegada, comprendidas por el Estado como entidades, formales e informales, para la enseñanza de formación artística especializada en estudiantes a lo largo de su etapa escolar. Forman parte de la opción educativa del Estado por fortalecer la educación artística como un elemento fundamental en la formación integral de las personas y están avaladas por el Seremi de Educación de cada región del país. Se encuentran distribuidas en cada una de las regiones de Chile, mayoritariamente en los sectores urbanos, siendo un aporte significativo para el desarrollo social y cultural de cada comuna (CNCA, 2015).

Estos centros escolares destacan por su enfoque educativo basado en la enseñanza de diferentes disciplinas artísticas tales como: artes visuales, artes musicales, artes teatrales, artes integradas, artes literarias, danza, cultura tradicional, otros. Estas disciplinas son integradas a la malla curricular común, razón por la cual se llevan a cabo los mismos 
parámetros de planificación, observación de clase, retroalimentación e incluso evaluación en relación con las demás disciplinas formales.

También, se aprecia un vínculo con el entorno mediante el desarrollo de actividades y presentaciones de elencos artísticos en actividades comunales o locales: concursos, festivales de artes o en actividades internas, en donde participa gran parte de los estudiantes y la comunidad educativa, y es invitada la comunidad local (CNCA, 2011). Aunque queda señalar que no todos estos centros escolares cuentan con elencos, de igual manera, los estudiantes participan en actividades específicas cuando se requiere.

Cabe señalar que, en relación con los recursos económicos y materiales la mayoría de los centros escolares poseen financiamiento mediante fondos concursables como el Fondo Nacional de Escuelas Artísticas (FNEA), el Programa Acciona, entre otros. Algunos centros escolares formales suelen contar con la Subvención Escolar Preferencial (SEP) para el desarrollo de las disciplinas artísticas. Y, en casos particulares, de las familias miembros de la comunidad educativa (CNCA, 2011).

Hoy, los centros escolares son testigos de los constantes cambios de la sociedad y, a su vez, los principales exponentes para el tratamiento de la diversidad (UNESCO, 2009), que "viene pareja a la crisis de la uniformidad y, en lo social, al desarrollo de los procesos democráticos que imponen el respeto a la diferencia" (Gairín, 2012, p. 8), puesto que la diversidad es un concepto que está y ha sido parte de muchos aspectos de la vida y, sobre todo, en lo que respecta al ámbito educativo (García y Delgado, 2017).

Situarse en la perspectiva de la diversidad obliga a repensar a los centros escolares la respuesta que puedan dar a las nuevas necesidades educativas: "supone poner énfasis en una nueva cultura, reformulando las propuestas de los centros y del sistema educativo" (Gairín, 1998, p. 245) para concebir procesos educativos más justos e igualitario para todos.

Para Cabrerizo y Rubio (2007), la atención a la diversidad involucra cualquier decisión o actuación que se determine en el ámbito educativo cuya finalidad sea atender a los estudiantes en su diversidad. En este sentido, implica "tener en cuenta las características individuales del alumnado para adoptar las estrategias y actuaciones educativas más efectivas y adecuadas en cada caso" (Cabrerizo y Rubio, 2007, p. 45) que respondan a sus diferentes necesidades educativas (Gómez, 2005).

De alguna manera, se trata de ofrecer un abanico de posibilidades para todos que se concreta en el desarrollo de prácticas inclusivas centradas en la organización y en la planificación de los recursos materiales y humanos, en donde se diseñan diferentes estrategias, se proporciona y se evalúa un currículum óptimo para que cada estudiante en su diversidad aprenda y se incentivan las relaciones colaborativas que promuevan el desarrollo de una auténtica cultura de la diversidad y mejora en la inclusión educativa (Martín y Mauri, 1996; Gairín, 1998; Echeita, 2006; Cabrerizo y Rubio, 2007).

La inclusión educativa puede ser comprendida desde diferentes perspectivas que involucran el reconocimiento al derecho de la educación y, la reestructuración de las culturas, las políticas y las prácticas educativas, aunque generalmente es relacionada con la apertura de los sistemas escolares hacia la atención a la diversidad (Escribano y Martínez, 2013; UNESCO, 2017).

De hecho, la UNESCO (2009) define la inclusión educativa como un proceso que permite "abordar y responder a la diversidad de las necesidades de todos los educandos a través de una mayor participación en el aprendizaje, las actividades culturales y comunitarias y reducir la exclusión dentro y fuera del sistema educativo" (p. 14). Esta 
posición, centrada en la apuesta de inclusión en los centros educativos, "implica cambios y modificaciones de contenidos, enfoques, estructuras y estrategias basados en una visión común y la convicción de que es responsabilidad del sistema educativo regular educar a todos los niños y niñas" (UNESCO, 2009, p. 14).

En otras palabras, se trata de una búsqueda constante por atender a la diversidad en términos dinámicos (Escribano y Martínez, 2013), ofreciendo a todos los estudiantes las mismas oportunidades educativas y el apoyo (curricular, personal, social, etc.), tanto para alcanzar el éxito académico y progreso personal, como para eliminar las barreras de acceso a la educación, la discriminación y la exclusión de los grupos más vulnerables (Ainscow, 2003; Echeita, 2006; Escarbajal et al., 2012; García, Massani y Bermúdez, 2016; Moreno, 2016; Cabada, 2017).

En un sentido más amplio, la inclusión implica la preparación de las personas "para vivir en una sociedad donde la diversidad no solo es legítima, sino que es apreciada como un valor" (Escarbajal et al., 2012, p. 140) y una experiencia educativa enriquecedora para el desarrollo de la dimensión humana (Parra, 2011) que enlaza claramente con una escuela abierta y comprometida con la realidad social y que está dispuesta a establecer mecanismos de mejora permanente (Gairín, 2012; Rodríguez y Gairín, 2015).

Al respecto, existen variedad de estrategias institucionales, tanto didácticas como organizativas, que pueden emplear los centros escolares a fin de facilitar la atención a la diversidad frente a la identificación de carencias y necesidades actuales para inclusión educativa.

Por su parte, Tomlison (2005) propone al docente como principal ejecutor de la actividad didáctica, quien debe comprender las diferentes capacidades, motivaciones e intereses de sus estudiantes. Junto con ello, desplegar una serie de estrategias didácticas que favorezcan el aprendizaje significativo de los estudiantes, así como su propia práctica pedagógica, tales como proponer y desarrollar acciones para la diversidad, anticiparse a los hechos, procurar planificar en relación con los ritmos de aprendizaje, organizar recursos y tiempos de la clase, entre otros. Por lo demás, asumir una actitud proactiva en la enseñanza y transformarse en un guía, capaz de responsabilizar a sus estudiantes con su aprendizaje, enseñándoles la manera de llevarlo a cabo, orientando la evaluación hacia el aprendizaje.

Por otro lado, Gairín (1998) y Gómez (2005) proponen a los docentes el desarrollo de otras estrategias didácticas para la atención a la diversidad focalizadas en los objetivos, contenidos, actividades de enseñanza-aprendizaje, metodología y evaluación en la programación curricular, que se resume en el siguiente cuadro 1: 


\section{Cuadro 1. Estrategias Didácticas}

\begin{tabular}{|c|c|}
\hline $\begin{array}{l}\text { OBJETIVOS Y } \\
\text { CONTENIDOS }\end{array}$ & $\begin{array}{l}\text { Los objetivos de clase y contenidos deben considerar los siguientes criterios: } \\
\text { - Relación con los contenidos, los ritmos de aprendizaje y conocimientos } \\
\text { previos. } \\
\text { - Priorización de objetivos. } \\
\text { - } \quad \text { Reterminar contenidos de la asignatura según los conocimientos de adaptaciones de contenios. } \\
\text { criterios de organización, selección, secuenciación y reducción de } \\
\text { contenidos. }\end{array}$ \\
\hline $\begin{array}{l}\text { ACTIVIDADES } \\
\text { DE ENSEÑANZA- } \\
\text { APRENDIZAJE }\end{array}$ & $\begin{array}{l}\text { - Diseñar actividades que permitan diferentes posibilidades de ejecución, } \\
\text { realización y dificultad. } \\
\text { - Organizar actividades de forma diferente, lineal y cíclica en función de } \\
\text { los diferentes ritmos de aprendizaje e intereses de los estudiantes. } \\
\text { Diseñar diversas actividades para trabajar un mismo contenido según } \\
\text { los ritmos de aprendizaje de los estudiantes. } \\
\text { - Proponer actividades de aprendizaje que permitan reforzar } \\
\text { conocimientos de los estudiantes. } \\
\text { - Planificar actividades de libre ejecución según los intereses de los } \\
\text { estudiantes. } \\
\text { - Promover actividades que permitan desarrollar en los estudiantes la } \\
\text { empatía y el respeto por los demás. } \\
\text { - Proponer actividades de aplicación práctica para la vida cotidiana. }\end{array}$ \\
\hline METODOLOGÍA & $\begin{array}{l}\text { - Realizar más clases prácticas que de explicación teórica. } \\
\text { - Tener en cuenta la disposición y el agrupamiento de los alumnos en el aula. } \\
\text { - Poner mayor énfasis en métodos de aprendizaje que favorezcan la } \\
\text { reflexión, la comunicación y el descubrimiento. } \\
\text { - Adecuar el lenguaje empleado en los materiales de estudio al nivel de } \\
\text { comprensión de los estudiantes, especialmente para los estudiantes con } \\
\text { necesidades educativas especiales. } \\
\text { - Proporcionar más tiempo a los estudiantes para la realización de tareas } \\
\text { en clases. } \\
\text { Trabajar algunos contenidos mediante su vinculación con otras áreas } \\
\text { de conocimiento. } \\
\text { Favorecer la utilización de variedad de recursos y materiales para que } \\
\text { los estudiantes puedan manipular y experimentar. }\end{array}$ \\
\hline EVALUACIÓN & $\begin{array}{l}\text { - Realizar adecuaciones en la evaluación para los estudiantes con N. E.E. } \\
\text { - Potenciar la autoevaluación del aprendizaje de los estudiantes. } \\
\text { - Realizar una evaluación inicial previo al proceso de enseñanza- } \\
\text { aprendizaje. } \\
\text { - Valorar la evaluación continua en el trabajo en clases, el interés y la } \\
\text { participación de los estudiantes. } \\
\text { Retroalimentar a los estudiantes los contenidos mínimos a estudiar } \\
\text { previo a una evaluación. } \\
\text { Utilizar variados instrumentos de evaluación (pruebas, trabajos, } \\
\text { entrevistas, etc.). }\end{array}$ \\
\hline
\end{tabular}

Estrategias didácticas para la atención a la diversidad (Fuente: Adaptado de Gairín, 1998; y Gómez, 2005). 
El énfasis está en mejorar la enseñanza explicitando diferentes estrategias didácticas en función del alumnado y su aprendizaje. No se trata de prevaler los contenidos, sino más bien, conviene pensar en introducir progresivamente diferenciaciones en los objetivos que se persiguen y en los contenidos que se requieren enseñar al alumnado, en concordancia con las actividades y metodologías propuestas en el proceso de enseñanza y aprendizaje. Así mismo, en relación con el proceso evaluativo del alumnado (Gairín, 1998; Gómez, 2005; Tomlison, 2005).

En este contexto, en donde la atención a la diversidad se torna imprescindible, la evaluación debe orientarse hacia el aprendizaje del alumnado, mayormente desde la evaluación formativa y formadora (Sanmartí, 2007), de modo que el profesorado pueda regular el proceso de enseñanza, estableciendo los cambios adecuados para ayudar a los estudiantes en la construcción de su propio aprendizaje, detectando sus dificultades y aciertos y fomentando el papel de estos en el proceso evaluador. La evaluación orientada al aprendizaje favorece la promoción de actividades auténticas y variadas, que aportan retroalimentación y conducen al desarrollo de la autoevaluación, la metacognición y la autorregulación (Padilla y Gil, 2008), constituyéndose en un elemento sumamente favorecedor para optimizar el aprendizaje. En este punto, es importante tener en cuenta que las posibilidades de aplicación de dichas estrategias didácticas para la atención a diversidad dependen de las variadas situaciones diferenciales que se puedan presentar en el aula y de las necesidades educativas individuales de los estudiantes (Gairín, 1998, Gómez, 2005; Tomlison, 2005).

En relación con las estrategias organizativas, Gairín (1998) destaca aquellas que aluden al sentido y funcionamiento de la cultura escolar de la diversidad. En primer lugar, la adecuación de los recursos del centro a las necesidades que una organización educativa planteada para todos, diseñando acciones acordes a su realidad contextual. En segundo lugar, reflejar el compromiso de una escuela para todos, es decir, registrar las ideas y las acciones para hacer efectiva la propuesta de inclusión a través de documentos institucionales (Proyecto Educativo Institucional (PEI), el Proyecto Curricular (PC) u otros). En tercer lugar, el aprendizaje organizativo mediante la realización de modificaciones de programas inclusivos en desarrollo, o al funcionamiento de la organización cuando surgen inconvenientes a mejorar.

Por otra parte, Álvarez et al. (2014) señalan otras estrategias organizativas para una escuela inclusiva que atiende a la diversidad. Destacamos aquellas que implican trabajar prioritariamente las actitudes (creencias), gestionar y adecuar los recursos con los que cuenta el centro, elaborar un Proyecto Educativo de centro inclusivo y trabajar colaborativamente, los cuales acentúan el trabajo en conjunto entre la comunidad educativa, y, por tanto, su cultura escolar de la diversidad.

También, se destacan iniciativas como las redes de colaboración con el entorno, diseñar proyectos de educación inclusiva y evaluar procesos. Estas estrategias convocan la necesidad de preparación de los centros escolares ante los cambios continuos de la sociedad, mediante la actualización permanente de sus procesos educativos y la ampliación de sus posibilidades de acción más allá de las que se puedan generar a nivel interno.

La participación del docente en la organización cobra gran sentido "porque de la percepción que tenga del alumnado y de sus procesos de aprendizaje va a depender los modelos educativos que ponga en juego" (Cabada, 2017, p. 180). Para ello, necesariamente, el centro debe facilitar los espacios y contribuir en estrategias promoviendo la accesibilidad 
de los docentes a los distintos recursos materiales del centro y espacios físicos para atender a la diversidad. Sobre este punto, Gairín (1998) y Gómez (2005) proponen estrategias organizativas orientadas al trabajo docente, que se resumen en el cuadro 2 :

Cuadro 2. Estrategias organizativas

\begin{tabular}{|c|c|}
\hline $\begin{array}{l}\text { ACCESIBILIDAD A } \\
\text { MATERIALES Y } \\
\text { ESPACIOS FÍSICOS }\end{array}$ & $\begin{array}{l}\text { - El centro escolar apoya el acceso de los docentes a distintos recursos } \\
\text { materiales y espacios físicos para atender a la diversidad. } \\
\text { Revisar con anterioridad los materiales o recursos didácticos que } \\
\text { utilizar en la clase. } \\
\text { - Utilizar materiales o recursos didácticos coherentes con la programación } \\
\text { de esta. } \\
\text { Organizar el espacio en el aula para favorecer la atención a la } \\
\text { diversidad de los estudiantes y su uso de manera flexible en las } \\
\text { actividades de clase. } \\
\text { Utilizar espacios distintos al aula para realizar las actividades al } \\
\text { interior del centro o fuera. }\end{array}$ \\
\hline $\begin{array}{l}\text { ORGANIZACIÓN } \\
\text { DE SISTEMAS DE } \\
\text { REFUERZO }\end{array}$ & $\begin{array}{l}\text { - Trabajar de manera coordinada y colaborativa con otros profesionales } \\
\text { para el trabajo específico con estudiantes (profesores de apoyo, } \\
\text { psicopedagogos, terapeuta ocupacional, entre otros). }\end{array}$ \\
\hline $\begin{array}{c}\text { GRUPOS DE } \\
\text { TRABAJO }\end{array}$ & $\begin{array}{l}\text { - Aplicar estrategias como agrupamientos flexibles, talleres, rincones, } \\
\text { grupos cooperativos, plan de trabajo, etc. para atender a la diversidad. } \\
\text { Formar grupos de aprendizaje de acuerdo con la heterogeneidad de } \\
\text { sus participantes. } \\
\text { Presentar modalidades flexibles de agrupación cuando se trata de } \\
\text { estudiantes que requieren una atención especial para el desarrollo de } \\
\text { actividades. }\end{array}$ \\
\hline
\end{tabular}

Estrategias organizativas para la atención a la diversidad (Fuente: Adaptado de Gairín, 1998; y Gómez, 2005).

La importancia de las estrategias organizativas recae en el planteamiento de las acciones y la actitud de los miembros para definirlas y llevarlas a cabo (Álvarez et al., 2014). Se transforman en la posibilidad de mejorar sus procesos, gestionar y organizar de otra manera su funcionamiento, asumiendo valores y creencias, basados en el compromiso social de la cultura escolar (Gairín, 2012; Rodríguez y Gairín, 2015).

De esta manera, pretendemos analizar las estrategias institucionales que se desarrollan en la Escuela de Cultura y Difusión Artística de Puerto Montt para atender a la diversidad puesto que el tipo de enseñanza y aprendizaje focalizado en el arte que se promueve tiene un valor trascendental para sus estudiantes y la comunidad a la que pertenecen, motivo por el cual se merece una respuesta desde la óptica de la inclusión educativa. Por eso, conviene analizar el tratamiento de la diversidad que se desempeña. 


\section{MÉTODO}

Para efectos de revisión bibliográfica y objetivo general del estudio se determinaron los siguientes objetivos específicos de investigación:

- Explorar las opiniones y valoraciones que tienen el equipo directivo y los docentes de la Escuela de Cultura y Difusión Artística de Puerto Montt sobre la atención a la diversidad.

- Identificar la variedad de estrategias institucionales que desarrolla la Escuela de Cultura y Difusión Artística de Puerto Montt para atender a la diversidad.

- Analizar las estrategias institucionales que influyen en la mejor inclusión educativa en la Escuela de Cultura y Difusión Artística de Puerto Montt.

Para el estudio se empleó una metodología de investigación de carácter mixto, descriptivo de prevalencia cualitativa, con el fin de obtener una perspectiva más profunda y amplia del fenómeno estudiado, lo que ha permitido una mejor "exploración y explotación" de los datos recabados (Hernández, Fernández y Baptista, 2016), ya que responde a un proceso más bien de carácter integral y holístico.

En relación con el enfoque mixto se trabajó con el estudio de caso por permitir situarnos en el marco contextual para analizar, desde una unidad de manera integrada, el problema y vislumbrar nuevas teorías. Para Hernández et al. (2016) el estudio de caso permite analizar en profundidad una unidad, responder al problema de investigación, comprobar una hipótesis y desarrollar o proponer teorías. El estudio de caso destaca por "su capacidad para generar premisas hipotéticas y orientar la toma de decisiones" (Álvarez y San Fabián, 2012 , p. 2), razón por la cual, estaría estrechamente vinculado a la teoría.

De acuerdo con Stake (2007): “el cometido real del estudio de caso es la particularización, no la generalización. Se toma un caso en particular y se llega a conocerlo bien” (p. 20). El estudio de caso de esta investigación se enmarca en la Escuela de Cultura y Difusión Artística de Puerto Montt.

La muestra fue intencional y estratifica mediante estrategia de muestreo básico para los métodos mixtos (Hernández et al., 2016) con el fin de segmentar la población en estratos. De esta manera, el criterio de selección consideró nivel de educación y años de experiencia de los participantes.

La muestra se constituye de 20 docentes de diferentes disciplinas y 3 miembros del equipo directivo, entre ellos, Director/a, Jefe/a de Unidad Técnica Pedagógica, Coordinador/a Artístico, siendo un total de 23 participantes miembros de la Escuela de Cultura y Difusión Artística de Puerto Montt.

Los instrumentos de recogida de información fueron: análisis documental, cuestionario y entrevista semiestructurada. En la tabla 1 se vincula cada instrumento con los objetivos del estudio y los participantes informantes: 
Tabla 1. Resumen instrumentos, objetivos de estudio y participante/objeto

\begin{tabular}{|l|l|c|}
\hline INSTRUMENTOS & \multicolumn{1}{|c|}{ OBJETIVOS } & PARTICIPANTE/OBJETO \\
\hline Análisis documental & $\begin{array}{l}\text { Identificar la variedad de estrategias } \\
\text { institucionales que desarrolla la Escuela } \\
\text { de Cultura y Difusión Artística de Puerto } \\
\text { Montt para atender a la diversidad. }\end{array}$ & $\begin{array}{l}\text { Proyecto Educativo } \\
\text { Institucional (PEI) } \\
\text { Proyecto Curricular (PC) }\end{array}$ \\
\hline Cuestionario & $\begin{array}{l}\text { Identificar la variedad de estrategias } \\
\text { institucionales que desarrolla la Escuela } \\
\text { de Cultura y Difusión Artística de Puerto } \\
\text { Montt para atender a la diversidad. }\end{array}$ & $\bullet \quad$ Docentes (20) \\
\hline Entrevista & $\begin{array}{l}\text { Explorar las opiniones y valoraciones } \\
\text { que tienen el equipo directivo y los } \\
\text { docentes de la Escuela de Cultura y } \\
\text { Difusión Artística de Puerto Montt sobre } \\
\text { la atención a la diversidad. }\end{array}$ & Equipo directivo (3) \\
\hline
\end{tabular}

(Fuente: elaboración propia)

Los documentos institucionales (PEI y PC) fueron solicitados a la dirección del centro escolar y también descargados de páginas web ministeriales. Se elaboró una matriz de análisis que consideró ocho dimensiones: análisis del contexto, compromiso con la diversidad, cultura de la diversidad, recursos, concreción curricular, estrategias organizativas, estrategias didácticas y propuestas de mejora.

El cuestionario dirigido a docentes fue elaborado a partir de la revisión teórica, que contempló preguntas de información general (género, edad, profesión, cargo actual, experiencia docente), 42 preguntas cerradas con escala de tipo Likert de 1 a 5, donde: Siempre: 5, Casi siempre: 4, A veces: 3, Casi Nunca: 2, Nunca: 1. En total fueron 3 ítems de respuesta (cultura escolar de la diversidad, estrategias organizativas y estrategias didácticas). También, el cuestionario incorporó tres preguntas abiertas.

La entrevista se constituyó en un guion de entrevista a partir de la revisión teórica y de los resultados del cuestionario. A partir del análisis Dexplis propuesto por Hernández et al. (2016), se identificaron variables que tuvieron respuestas confusas, para luego, profundizar en la entrevista. De esta manera, se diseñó una entrevista semiestructura dirigida al equipo directivo, que se organizó a partir de tres temáticas. En primer lugar, aspectos generales (descripción personal, formación académica, trayectoria profesional), en segundo lugar, cultura escolar de la diversidad y, por último, estrategias organizativas. Estas dos últimas temáticas se abordaron en 10 preguntas.

El cuestionario y la entrevista fueron sometidos a la validación de 5 jueces expertos en el tema objeto de estudio según criterios de univocidad, pertinencia e importancia. Para el análisis cuantitativo de datos de la validación del cuestionario se determinó un porcentaje de corte de $75 \%$ para los criterios de univocidad y pertinencia y la media de 3,5 en importancia. En el caso del análisis cualitativo de datos, tanto en el cuestionario como en la entrevista se tomó en consideración las sugerencias de los expertos y luego se modificaron los instrumentos en términos de redacción y separación de ideas. 
Para el tratamiento de la información del análisis documental, se elaboró una matriz de información de los planteamientos institucionales. En el caso del cuestionario, se realizó análisis descriptivo de frecuencia, el cual se apoyó del Programa Estadístico SPSS mediante el uso de tablas de frecuencias que dieron a conocer el número y porcentaje de cada valor observado de una variable. También, se realizó una matriz para análisis preguntas abiertas. Por último, para la obtención de datos de las entrevistas se empleó el Programa Atlas-ti, a partir de categorías de análisis de codificación, que se apoyó con el diseño de una matriz de análisis según dimensiones.

En el análisis de los resultados, se optó por la técnica de triangulación de datos (Hernández et al., 2016) para validar la investigación y enriquecer los resultados, la cual permitió extraer, verificar y comparar la información obtenida de cada instrumento, estableciendo con ello cinco dimensiones para el análisis que corresponden a: la valoración de la diversidad desde la inclusión educativa, las concepciones sobre la atención a la diversidad, los propósitos para la atención a la diversidad, las estrategias didácticas y las estrategias organizativas para la atención a la diversidad.

\section{RESULTADOS}

\subsection{VALORACIÓN DE LA DIVERSIDAD DESDE LA INCLUSIÓN EDUCATIVA}

Con respecto a la valoración de la diversidad desde la inclusión educativa encontramos que, en la Escuela de Cultura y Difusión Artística de Puerto Montt la inclusión educativa forma parte de un proyecto educativo que valora y atiende la diversidad, puesto que genera cambios en su enfoque y estructura tanto a nivel curricular como organizativo del centro.

En la siguiente tabla 2, que resume los datos obtenidos del cuestionario mediante tablas de frecuencia, se observa una mayor tendencia hacia la promoción de una cultura escolar de la diversidad:

Tabla 2. Resumen dimensión cultura escolar de la diversidad

\begin{tabular}{|l|c|}
\hline \multicolumn{1}{|c|}{ Pregunta } & Porcentaje \\
\hline $\begin{array}{l}\text { 1. El centro escolar promueve la comprensión de las diferencias de los estudiantes } \\
\text { respecto a su ámbito cognitivo. }\end{array}$ & $85 \%$ \\
\hline $\begin{array}{l}\text { 2. El centro escolar promueve la comprensión de las diferencias de los estudiantes } \\
\text { respecto a su ámbito afectivo. }\end{array}$ & $85 \%$ \\
\hline $\begin{array}{l}\text { 3. El centro escolar promueve la comprensión de las diferencias de los estudiantes } \\
\text { respecto a su ámbito relacional. }\end{array}$ & $75 \%$ \\
\hline $\begin{array}{l}\text { 4. El centro escolar se preocupa por la distinción entre la educación formal y } \\
\text { especializada según las capacidades, intereses y motivaciones. }\end{array}$ & $80 \%$ \\
\hline
\end{tabular}




\begin{tabular}{|l|c|}
\hline $\begin{array}{l}\text { 5. El centro escolar promueve el trabajo colaborativo entre los miembros de la } \\
\text { comunidad educativa para identificar necesidades educativas de los estudiantes y } \\
\text { plantear estrategias para atender a la diversidad. }\end{array}$ & $75 \%$ \\
\hline $\begin{array}{l}\text { 6. Las estrategias o actuaciones de intervención de la diversidad son descritos en } \\
\text { documentos institucionales del centro escolar. }\end{array}$ & $85 \%$ \\
\hline $\begin{array}{l}\text { 7. El centro escolar apoya el acceso de los docentes a distintos recursos materiales } \\
\text { y espacios físicos para atender a la diversidad. }\end{array}$ & $55 \%$ \\
\hline
\end{tabular}

Fuente: elaboración propia.

Además, este compromiso con la atención a la diversidad también se condice con lo declarado en el Proyecto Educativo Institucional (PEI), que tiene por misión formar ciudadanos íntegros, tanto en conocimientos y habilidades artísticas como en valores para relacionarse con los demás y respetar la diversidad, desplegando con ello una serie de actuaciones educativas que involucran la participación de la comunidad educativa.

\subsection{CONCEPCIONES SOBRE LA ATENCIÓN A LA DIVERSIDAD}

En lo que respecta a las concepciones sobre la atención a la diversidad encontramos diferentes opiniones y valoraciones por parte de los docentes y el equipo directivo.

La mayoría de los docentes informantes se inclinaron a señalar que el trabajo con la diversidad implica un aprendizaje personal y profesional:

Es una experiencia enriquecedora que sirve para ser más humano (CD4).

Ha representado un desafío y un continuo aprendizaje (CD6).

Una oportunidad y un desafío. Lo primero es que me ayuda a desarrollar la colaboración y tolerancia del grupo en su trabajo. La música coral trabaja con todos y para un mismo resultado. Por lo que el avance de todos es el objetivo para tener un final de cada uno de los trabajos lo mejor que se pueda, buscando cada día mejorar técnicas para avanzar todos juntos (CD7).

Es significativa, puesto que estás en una constante búsqueda, me refiero a buscar el material que ayude a mejorar su aprendizaje y de todos (CD14).

El aprendizaje profesional se vincularía a la mejora de prácticas pedagógicas, el aprendizaje de los estudiantes, la relación con estos y con los demás profesionales del centro escolar.

Otro grupo docente valora la diversidad desde la influencia que tiene en el arte. De esta manera exponen que es:

La posibilidad de enriquecer el producto artístico (CD12).

Significa diferentes maneras de ver la música, de pensarlo (CD16).

Es una experiencia transformadora y enriquecedora (...) enseñar en un espacio artístico y donde se valora de manera concreta las aptitudes creativas de los estudiantes (CD18). 
Por último, se reconocen otras concepciones sobre la diversidad, en donde algunos docentes señalan que:

Ha sido gratificante, ya que no debe existir discriminación alguna y menos en el aula (CD1).

Tener que cambiar el paradigma de la enseñanza, ya que todos tienen las mismas posibilidades; mismas oportunidades para aprender (CD3).

A raíz de las entrevistas al equipo directivo se obtuvo que la atención a la diversidad en el contexto educativo es atender a todas las necesidades de los estudiantes:

es atender a todos los estudiantes, en qué sentido. En que hay estudiantes que presentan asistencia de tipo cognitiva, cultural, social, en género, ciertos impedimentos físicos, etc. Y la atención a la diversidad significa que uno tiene que estar pendiente de todos estos niños, tiene que (...) hacer que estos niños puedan insertarse ... en el ámbito educacional y también social a través de sus diferentes estilos, formas cierto de aprendizaje (EED1).

También podemos relacionar de lo expresado por un miembro del equipo directivo que la diversidad en el contexto educativo:

Es atender a grupos de personas diferentes, con diferentes ideologías, ya que los niños están en formación, van cambiando sus pensamientos con diferentes culturas y también con diferentes orientaciones sexuales y con necesidades educativas especiales también (EED2).

A esto se añade otra idea que tiene que ver con la diversidad como derecho humano, ya que mencionan que la diversidad es un proceso natural de la persona:

Creo que es un derecho humano, es un elemento sumamente esencial en la construcción de cualquier ser humano (EED3).

\subsection{PROPÓSITOS PARA LA ATENCIÓN A LA DIVERSIDAD}

En lo que se refiere a los propósitos para la atención a la diversidad, el equipo directivo considera, en primer lugar, que es importante entender y atender a la diversidad dado los beneficios que trae consigo para el aprendizaje integral de sus estudiantes. De esta manera, se exponen dos razones:

para nosotros es sumamente importante debido a que somos una escuela que tiene características distintas, que a través del arte nosotros desarrollamos habilidades y destrezas que no se desarrollan comúnmente en una sala de clase (EED1).

la educación artística, o el arte vinculado, orientado a la formación de un ser humano es un elemento esencial. Construye un ser integral, desarrolla áreas tal vez un poco más dormidas en un niño, en un adulto incluso (EED3). 
Por otra parte, la importancia de atender a la diversidad se constituye a raíz de comprender las diferentes necesidades educativas de los estudiantes según sus estilos e intereses en el aprendizaje:

es importante porque eso también, va variando cómo ellos aprenden, sus distintos estilos de aprendizaje y los intereses que ellos también tienen. Por lo tanto, esa son cosas que se debe tomar en cuenta, en el momento de realizar las clases o de preparar el material para que sea algo que a ellos le llame la atención y participen activamente (EED2).

En cuanto a los propósitos para atender a la diversidad, se exponen diferentes perspectivas. En primer lugar, se defiende que el propósito sería preparar a los estudiantes para la vida:

nosotros lo que pretendemos es formar personas para la vida. Esa es la mayor preocupación y ese es el plus que tiene este establecimiento de preparar personas para la vida (EED1).

Este propósito se basa en lograr la formación integral de los estudiantes, quienes deben aprender no solo conocimientos, sino que también actitudes y valores sociales, para convivir, aprender y respetar a los demás:

Para formar personas íntegras, ya. Una escuela es un centro donde se reúnen distintos tipos de personas que se encuentran en la sociedad. Por lo tanto, es importante que ellos aprendan a convivir y que se formen a través de valores como la tolerancia, el respeto, esa es la finalidad y además de que ellos creen competencias que sean importantes para la vida (EED2).

En relación con lo anterior, el equipo directivo considera que la adquisición de conocimientos y valores de ese tipo implicaría que los estudiantes puedan formar parte de un grupo, evitando la exclusión social y favoreciendo el sentido de pertenencia:

Estar insertos en un grupo, es que ellos hoy en día y mañana, puedan, cierto, tener opinión y puedan sentirse personas y ser parte de una sociedad que muchas veces los deja de lado. Ese el plus que nosotros tenemos como establecimiento más allá de que se nos pueda exigir la parte académica que también es importante (EED1).

No estigmatizándolo ni dejándolo de lado, sino que sea parte del grupo curso, que sea parte del establecimiento y que tenga un sentido de pertenencia con el establecimiento (EED1).

Por otro lado, otro miembro del equipo directivo menciona que el propósito de atender a la diversidad tiene relación con el desarrollo del arte, específicamente, formar artistas:

la orientación es entregar herramientas artísticas que contribuyan a un desarrollo intermedio, avanzado en cuanto a, al desempeño artístico de los estudiantes, es decir que los chiquillos que estudian en nuestro establecimiento salgan con elementos que 
les puedan servir como para continuar estudios superiores (EED3).

Y obviamente apuntando a formar seres integrales, desarrollando aspectos más integrales de la personalidad de cada estudiante (EED3).

De esta manera, se entendería que adquirir conocimientos y habilidades en el arte, es también un aspecto importante para la formación integral de los estudiantes.

\subsection{LAS ESTRATEGIAS DIDÁCTICAS PARA LA ATENCIÓN A LA DIVERSIDAD}

En lo que se refiere a las estrategias didácticas para la atención a la diversidad se obtuvo que los docentes desarrollan diferentes estrategias orientadas a mejorar y enriquecer el proceso de enseñanza y aprendizaje de sus estudiantes focalizando la respuesta educativa en los objetivos, contenidos, actividades, metodologías y evaluación de su programación curricular. Esto se observan en la siguiente tabla 3, que resumen los datos obtenidos en el análisis descriptivo de las tablas de frecuencia del cuestionario:

Tabla 3. Estrategias didácticas

\begin{tabular}{|l|c|}
\hline \multicolumn{1}{|c|}{ Pregunta } & Porcentaje \\
\hline $\begin{array}{l}\text { Se establecen objetivos de clase de acuerdo con los contenidos, los ritmos de } \\
\text { aprendizaje y conocimientos previos de los estudiantes. }\end{array}$ & $90 \%$ \\
\hline La evaluación inicial previo al proceso de enseñanza-aprendizaje. & $90 \%$ \\
\hline $\begin{array}{l}\text { La evaluación continua valora el trabajo en clases, el interés y la participación de los } \\
\text { estudiantes. }\end{array}$ & $90 \%$ \\
\hline $\begin{array}{l}\text { La retroalimentación con los estudiantes y los contenidos mínimos previos a una } \\
\text { evaluación. }\end{array}$ & $90 \%$ \\
\hline El uso de variados instrumentos de evaluación (pruebas, trabajos, entrevistas, etc.). & $90 \%$ \\
\hline Las adecuaciones en la evaluación para los estudiantes con N.E.E. & $90 \%$ \\
\hline Se potencia la autoevaluación del aprendizaje de los estudiantes. & $85 \%$ \\
\hline $\begin{array}{l}\text { Se diseñan actividades que permitan diferentes posibilidades de ejecución, } \\
\text { realización y dificultad. }\end{array}$ & $85 \%$ \\
\hline $\begin{array}{l}\text { Se diseñan diversas actividades para trabajar un mismo contenido según los ritmos } \\
\text { de aprendizaje de los estudiantes. }\end{array}$ & $85 \%$ \\
\hline $\begin{array}{l}\text { Se proponen actividades de aprendizaje que permiten reforzar conocimientos de los } \\
\text { estudiantes. }\end{array}$ & $80 \%$ \\
\hline $\begin{array}{l}\text { Se promueven actividades que permiten desarrollar en los estudiantes la empatía y } \\
\text { el respecto por los demás. }\end{array}$ & $85 \%$ \\
\hline $\begin{array}{l}\text { Para la realización de la clase se tiene en cuenta la disposición y el agrupamiento de } \\
\text { los alumnos en el aula. }\end{array}$ & $\begin{array}{l}\text { Se pone mayor énfasis en métodos de aprendizaje que favorezcan la reflexión, la } \\
\text { comunicación y el descubrimiento. }\end{array}$ \\
\hline
\end{tabular}




\begin{tabular}{|l|l|}
\hline $\begin{array}{l}\text { El lenguaje empleado en los materiales de estudio se adecua al nivel de comprensión } \\
\text { de los estudiantes, especialmente para los estudiantes con necesidades educativas } \\
\text { especiales. }\end{array}$ & $80 \%$ \\
\hline $\begin{array}{l}\text { Se realizan adaptaciones de contenidos curriculares de acuerdo con criterios de } \\
\text { organización, selección, secuenciación y reducción de contenidos. }\end{array}$ & $75 \%$ \\
\hline $\begin{array}{l}\text { Se organizan actividades de forma diferente, lineal y cíclica en función de los } \\
\text { diferentes ritmos de aprendizaje e intereses de los estudiantes. }\end{array}$ & $75 \%$ \\
\hline $\begin{array}{l}\text { Los contenidos de la asignatura se determinan según los conocimientos previos de } \\
\text { los estudiantes. }\end{array}$ & $70 \%$ \\
\hline Las actividades propuestas tienen aplicación en la vida cotidiana. & $70 \%$ \\
\hline Se realizan más clases prácticas que de explicación teórica. & $70 \%$ \\
\hline Se proporciona más tiempo a los estudiantes con necesidades educativas especiales. & $65 \%$ \\
\hline $\begin{array}{l}\text { Algunos contenidos son trabajados mediante su vinculación con otras áreas del } \\
\text { conocimiento. }\end{array}$ & $65 \%$ \\
\hline $\begin{array}{l}\text { Se emplean técnicas de enseñanza específicas como enseñanza tutorizada } \\
\text { monografías, propuestas de investigación, trabajo colaborativo, etc. según las } \\
\text { necesidades educativas de los estudiantes. }\end{array}$ & $60 \%$ \\
\hline
\end{tabular}

Fuente: elaboración propia.

Es posible identificar el trabajo con la diversidad según los ritmos de aprendizaje de los estudiantes, para lo cual se proponen objetivos y contenidos que permitan optimizar el aprendizaje. De igual manera, reforzar los conocimientos previos de los estudiantes se transforma en una práctica mayormente habitual, tanto para la propuesta de objetivos y contenidos, como para la evaluación.

Una estrategia didáctica que sobresale se relaciona con el refuerzo hacia los estudiantes con N.E.E., dado que hay una preocupación por adecuar el lenguaje en los materiales de trabajo, proporcionar más tiempo en las actividades y realizar adecuaciones en las evaluaciones. Esto se comprende como consecuencia del trabajo colaborativo con otros profesionales del centro que son parte del Programa de Integración Escolar.

Cabe señalar que existe interés en proponer actividades de clases que contribuyan a mejorar las relaciones sociales entre todos los estudiantes, como es el caso de realizar actividades que promuevan la empatía y respeto por los demás. Junto a esto, se destaca que las actividades de clase tengan una aplicación práctica para la vida cotidiana, que se corresponde con los objetivos de la misión y visión del PEI del centro escolar, y que, a su vez, es corroborado por los miembros del equipo directivo.

Otras estrategias didácticas para la atención a la diversidad que desarrollan los docentes suelen ser:

- Proporcionar orientación para la continuidad de estudios.

- Apoyar la clase con material audiovisual.

- Trabajar con material de laboratorio.

- $\quad$ El arte como estrategia. 
En el caso de la orientación para la continuidad de estudios, es importante subrayar que aparece señalada en el PEI del centro escolar, y luego es corroborada por un miembro del equipo directivo (EED3) en la entrevista, quien hace hincapié en que la intencionalidad de los docentes para motivar a los estudiantes a continuar estudios estaría relacionada con la enseñanza artística, puesto que se les proporciona orientación y enseñanza de habilidades y destrezas mediante el vínculo de los contenidos curriculares del área formal y el área artística. De esta manera, destaca el interés de los docentes por emplear las artes para el trabajo con la diversidad.

\subsection{LAS ESTRATEGIAS ORGANIZATIVAS PARA LA ATENCIÓN A LA DIVERSIDAD}

Las estrategias organizativas para la atención a la diversidad son elaboradas y ejecutadas tanto por el equipo directivo como docentes del centro escolar.

Respecto a los resultados obtenidos del análisis documental y las entrevistas, se identifican las siguientes estrategias organizativas:

- Construcción conjunta del Proyecto Educativo.

- Evaluación y seguimiento del PEI.

- Redes de colaboración.

- Destinar las ocho horas lectivas de Jornada Escolar Completa (JEC) para la enseñanza artística.

Estas estrategias organizativas responden al sentido y funcionamiento de la cultura escolar de la diversidad, los cuales involucran el trabajo en conjunto de los miembros de la comunidad educativa a partir de diferentes instancias y espacios de reflexión que contribuyan a diseñar estrategias de atención a la diversidad y plasmarlas en los documentos institucionales del centro escolar.

Cabe señalar que, tras las entrevistas se ha podido identificar otros documentos institucionales elaborados mayormente por el equipo directivo y los profesionales del Programa de Integración Escolar (PIE), los cuales corresponden a planificaciones y entrevistas para el seguimiento del trabajo que se realiza con los estudiantes con N.E.E. y documentos de registro de las conversaciones que se tienen con los docentes y psicóloga del PIE, los cuales han sido necesarios en la toma de decisiones para el trabajo con la diversidad con objeto de que los estudiantes tengan un aprendizaje efectivo y de calidad.

Las redes de colaboración contribuyen a establecer relaciones de apoyo y coordinación con otras instituciones o agentes externos con el objetivo de generar distintas instancias de aprendizaje para los estudiantes, tanto a nivel pedagógico como de orientación hacia la continuidad de estudios.

Por otra parte, se observa que los docentes del centro escolar desarrollan otras estrategias organizativas, las cuales se exponen en la tabla 4: 
Tabla 4. Estrategias organizativas

\begin{tabular}{|l|c|}
\hline \multicolumn{1}{|c|}{ Pregunta } & Porcentaje \\
\hline $\begin{array}{l}\text { Los materiales o recursos didácticos que utilizar en la clase son coherentes con la } \\
\text { programación de esta. }\end{array}$ & $95 \%$ \\
\hline $\begin{array}{l}\text { Los materiales o recursos didácticos que utilizar en la clase se revisan con } \\
\text { anterioridad. }\end{array}$ & $90 \%$ \\
\hline $\begin{array}{l}\text { Se organiza el espacio en el aula para favorecer la atención a la diversidad de los } \\
\text { estudiantes y su uso de manera flexible en las actividades de clase. }\end{array}$ & $85 \%$ \\
\hline $\begin{array}{l}\text { Aplicación de agrupamientos flexibles, talleres, rincones, grupos cooperativos, } \\
\text { plan de trabajo, etc. para atender a la diversidad. }\end{array}$ & $85 \%$ \\
\hline $\begin{array}{l}\text { Coordinación docente y colaboración con otros profesionales para el trabajo } \\
\text { específico con estudiantes (profesores de apoyo, psicopedagogos, terapeuta } \\
\text { ocupacional, entre otros). }\end{array}$ & $80 \%$ \\
\hline $\begin{array}{l}\text { Los docentes presentan modalidades flexibles de agrupación cuando se trata de } \\
\text { estudiantes que requieren una atención especial para el desarrollo de actividades. }\end{array}$ & $80 \%$ \\
\hline $\begin{array}{l}\text { Se utiliza espacios distintos al aula para realizar las actividades al interior del } \\
\text { centro o fuera. }\end{array}$ & $70 \%$ \\
\hline $\begin{array}{l}\text { Formación de grupos de aprendizaje de acuerdo con la heterogeneidad de } \\
\text { estudiantes. }\end{array}$ & $65 \%$ \\
\hline
\end{tabular}

Fuente: elaboración propia.

Dichas estrategias organizativas se desarrollan para responder a la programación curricular según las necesidades educativas de los estudiantes.

El uso de recursos tales como materiales didácticos, del espacio dentro y fuera del centro contribuyen a potenciar el desarrollo de la práctica pedagógica del docente mediante la planificación previa de los recursos a utilizar en el proceso de enseñanza y aprendizaje.

La actividad colaborativa con otros profesionales es una práctica que se realiza constantemente en el centro escolar. Los primeros resultados obtenidos del cuestionario a docentes, nos señaló que el $80 \%$ de ellos generalmente trabaja colaborativamente para atender las necesidades de los estudiantes que requieren un apoyo específico. De acuerdo con un miembro del equipo directivo (EED3), esta actividad correspondería a una necesidad de los docentes por aprender estrategias que les permitan ofrecer a sus estudiantes una enseñanza adaptada a sus necesidades educativas, sobre todo de aquellos con N.E.E. Por lo demás, el trabajo colaborativo cobra gran importancia para la dirección del centro escolar, puesto que se destinan más horas para que los docentes la desarrollen.

Por último, se identificaron otras estrategias organizativas que realiza el centro escolar, las cuales son:

- Variedad de talleres artísticos y deportivos.

- Perfeccionamiento de todos los funcionarios. 
- Programa de Integración Escolar (PIE).

- Atención individualizada.

- Integración curricular.

- Trabajar con redes sociales.

A nivel organizativo se incluirían las estrategias relacionadas con el perfeccionamiento de funcionarios y el trabajo con redes sociales. En el primer caso, no se logró profundizar en el tipo de perfeccionamiento producto de la poca información sobre el tema en particular. En el segundo caso, el trabajo con las redes sociales aludiría al uso de las tecnologías de información y comunicación (TICs), el cual sería empleado para la difusión de las distintas actividades que desarrolla el centro escolar.

Las demás estrategias organizativas responden a la necesidad de intervenir en el ámbito curricular, como es el caso de proponer variedad de talleres, la atención individualizada, la integración curricular y el Programa de Integración Escolar (PIE). Estas se orientan al trabajo específico con los estudiantes según sus distintas necesidades educativas en cuanto a sus capacidades, intereses y motivaciones, sobre todo en lo que respecta al proyecto educativo basado en la enseñanza artística.

\section{DISCUSIÓN Y CONCLUSIONES}

En relación con las opiniones y valoraciones sobre la atención a la diversidad, se identifican diferentes concepciones entre los miembros del centro escolar. Al respecto, podemos concluir que, desde una perspectiva más general, el equipo directivo se inclina por mantener que la diversidad es un concepto que forma parte del sistema educativo y, por lo tanto, se transforma en deber por comprender y atender a las diferencias de los estudiantes (Aisncow, 2003; UNESCO, 2009; Escribano y Martínez, 2013; UNESCO, 2017). Según Cabrerizo y Rubio (2007) este aspecto responde a uno de los primeros criterios para que un centro escolar responda a la diversidad, ya que reconoce que los estudiantes poseen capacidades, intereses y motivaciones diferentes frente al aprendizaje. Por una parte, para la mayoría de los docentes, la atención a la diversidad se relaciona con un aprendizaje personal y profesional, frente a lo cual es posible evidenciar una postura a raíz de la experiencia con el trabajo de la diversidad (Escarbajal et al., 2012; Cabada, 2017).

Por otra, el equipo directivo tiene una valoración positiva sobre la atención a la diversidad y la considera importante para que las artes puedan generar mayores destrezas y habilidades en los estudiantes (Graeme, 2003; CNCA, 2011).

La atención a la diversidad en el centro estudiado ha permitido considerar el trabajo de formar estudiantes integrales desde la comprensión y el respeto hacia la diversidad. Esto se ha transformado en una meta para lograr aprendizaje en conocimientos y valores para la vida. Lo sostiene la idea de que esta preparación contribuirá a evitar la exclusión de los grupos y favorecerá el sentido de pertenencia de sus estudiantes (Moreno, 2016). Así, para el equipo directivo, la enseñanza artística es un medio esencial para abordar la diversidad en una perspectiva de formación integral de los estudiantes (Palacios, 2006; Jiménez, 2011; CNCA, 2011).

El centro escolar ha dispuesto de una programación curricular que posibilita la distinción entre la educación formal y especializada, al proporcionar a los estudiantes la oportunidad de acceder y aprender de diferentes disciplinas, tanto formales como artísticas 
en favor de sus diferentes capacidades, intereses y motivaciones por el aprendizaje. También cabe subrayar la priorización que se hace de las medidas basadas en la enseñanza adaptativa (Martín y Mauri, 1996). Estas hacen referencia a la intervención de los docentes en el proceso de enseñanza y aprendizaje.

En relación con el segundo objetivo podemos concluir que en la Escuela de Cultura y Difusión Artística de Puerto Montt se articulan distintas estrategias institucionales para atender a la diversidad de sus estudiantes, específicamente, son estrategias didácticas y organizativas que coinciden con las ya propuestas por Gairín (1998), Gómez (2005), Tomlison (2005) y Álvarez et al. (2014).

En relación con el diseño de las estrategias institucionales, concluimos que las mismas se sostienen en el planteamiento de criterios para atender a la diversidad en cuanto a las diferentes capacidades, intereses y motivaciones de los estudiantes. Se concretan en el desarrollo de prácticas inclusivas centradas en la organización y la planificación de los recursos humanos y materiales con que se cuentan y puede generar el centro escolar (Cabrerizo y Rubio, 2007).

Es así como se evidencia la comprensión y la valoración de las diferencias de los estudiantes frente a la creciente conformación de una cultura escolar de la diversidad, en donde denota cierto grado de compromiso institucional con la diversidad (Gairín, 1998; Gairín, 2012; Álvarez et al., 2014).

De acuerdo con el tercer objetivo, las estrategias institucionales que contribuyen a la mejora en la inclusión educativa son aquellas estrategias didácticas y organizativas que permiten gestionar los recursos del centro, fortalecer la práctica pedagógica, establecer diferentes modalidades de trabajo entre los miembros y promover el aprendizaje personal y colectivo de los estudiantes, en la búsqueda de mejoras institucionales permanentes.

En lo que se refiere a las estrategias didácticas para la atención a la diversidad destacan aquellas referidas a los objetivos y contenidos, en donde se establecen objetivos de clase de acuerdo con los contenidos, ritmos de aprendizaje y conocimientos previos de los estudiantes y se realizan adaptaciones de los contenidos curriculares según criterios de organización, selección, secuenciación y reducción de contenidos. En el caso de las actividades de enseñanza-aprendizaje, las más empleadas por los docentes son proponer diversas actividades para trabajar un mismo contenido y actividades de reforzamiento. Por otra parte, la mayoría de las actividades que proponen a sus estudiantes tienen relación con promover la empatía y el respeto por los demás, y otras de aplicación práctica para la vida. Según Gómez (2005), esta última es una de las finalidades de la atención a la diversidad, ya que se espera que al finalizar la etapa escolar los estudiantes posean conocimientos y actitudes que les permitan desenvolverse a los nuevos desafíos en la vida cotidiana.

En lo que respecta a las estrategias didácticas enfocadas en la metodología, por parte de los docentes se destaca la utilización de un lenguaje adecuado para la comprensión de los estudiantes con N.E.E. En relación con la evaluación, el énfasis está en la retroalimentación, la utilización de variados instrumentos de evaluación, la realización de adecuaciones para los estudiantes con N.E.E. y la búsqueda por potenciar la autoevaluación de los estudiantes.

En este sentido, se aprecia que las diferentes estrategias empleadas buscan la comprensión de los distintos ritmos de aprendizaje de los estudiantes. Siguiendo a Tomlison (2005), los docentes asumirían un rol de organizadores de oportunidades de aprendizaje.

Sin embargo, hay que señalar que, si bien, se destaca que los objetivos de clase se establecen de acuerdo con los contenidos, los ritmos de aprendizaje y conocimientos 
previos de los alumnos, en lo que se refiere a priorizar objetivos según el aprendizaje de los estudiantes, este último no suele ser representativo. En este sentido, surgen contradicciones, puesto que en ambos casos los objetivos de clase han de ser resueltos según lo logrado por el estudiante, lo cual favorece el desarrollo del aprendizaje significativo de los mismos. Por lo mismo, no se comprende que exista una diferencia considerable respecto al uso de estas estrategias didácticas.

Por otro lado, la mayoría de las estrategias referidas a la comprensión de los intereses de los estudiantes no se desarrollan con frecuencia, como en el caso de proponer actividades de libre ejecución. Al respecto, se observa la atención a los intereses de los estudiantes cuando estos pueden realizar autoevaluaciones de su aprendizaje. En este sentido, sería importante considerar que los docentes desarrollen también otras estrategias que permitan atender a los intereses. Con ello, es posible que los estudiantes se transformen en participantes activos de su propio aprendizaje (Tomlison, 2005).

En el caso de las estrategias organizativas, resaltan algunas como: la construcción conjunta del Proyecto Educativo, evaluación y seguimiento del PEI, redes de colaboración, destinación de horas lectivas para la enseñanza artística y el Programa de Integración Escolar.

Entre las estrategias organizativas realizadas por los docentes del centro escolar, se hacen notar aquellas que tienen relación con el uso de variados materiales didácticos revisados con anterioridad y coherentes con la programación curricular, la organización del espacio del aula, el trabajo colaborativo con otros profesionales del centro y las modalidades flexibles de agrupamiento de los estudiantes de acuerdo con la heterogeneidad de estos. Estas estrategias permiten evidenciar la preocupación por responder y valorar la diversidad de sus estudiantes, puesto que se comprende que existe una programación de la clase previamente definida, organizada y enriquecida con el uso de diferentes recursos que fortalecerían el proceso de enseñanza y aprendizaje (Gairín, 1998; Gómez, 2005).

Para finalizar, podemos mencionar que en el centro escolar analizado se puede identificar la existencia de autonomía institucional para llevar a cabo cambios o modificaciones a nivel curricular y organizativo con el propósito de atender a la diversidad y mejorar los procesos educativos (Echeita, 2006; Cabrerizo y Rubio, 2007; Rodríguez y Gairín, 2015). Mayormente se vislumbra frente a la destinación de horas de libre disposición para desarrollar distintos talleres de disciplinas artísticas, y propuestas directivas. El hilo conductor es el trabajo colaborativo de los profesionales, la planificación conjunta y la creación de instrumentos institucionales para evaluar procesos y proponer mejoras.

Con todo, la dificultad más acentuada que tiene el centro escolar para progresar en su esfuerzo por atender a la diversidad tiene que ver con la implantación de la cultura de la diversidad. Si bien es cierto que existe interés de los miembros por atender a la diversidad, también lo es que faltan lineamientos entre las prácticas colaborativas que se ponen en marcha para que sea efectiva. Sin ello, se corre el peligro de caer en el trabajo individualizado entre el equipo directivo y docentes.

En esta misma línea, los miembros del centro escolar son conscientes de que conviene una mayor implicación de la comunidad educativa, y en particular de las familias. También, la consecución de más recursos que contribuyan a mejorar la infraestructura del centro escolar, proporcionando salas de clases para el trabajo con disciplinas artísticas, la incorporación de laboratorios y sala de computación o materiales didácticos adaptados, también se ve como un elemento destacado que contribuiría al propósito perseguido. 
Más aún cuando, la previsión de recursos específicos es significativa para el trabajo con la diversidad, ya que supone que los centros escolares diseñen propuestas a partir de los recursos propios y que estén contextualizados a su realidad escolar.

\section{REFERENCIAS BIBLIOGRÁFICAS}

Ainscow, M. (octubre de 2003). Desarrollo de Sistemas Educativos Inclusivos. Ponencia llevada a cabo en el Congreso La respuesta a las necesidades educativas especiales en una escuela vasca inclusiva, San Sebastián, España.

Álvarez, C. y San Fabián, J. (2012). La elección del estudio de caso en investigación educativa. Gazeta de Antropología, 28(1), 1-12.

Álvarez, J., Grau, S. y Tortosa, M. (2014). Estrategias organizativas para una escuela inclusiva. International Journal of Developmental and Educational Psychology, 7(1), 53-61.

Cabada, J. (2017). Los caminos de la inclusión y los peligros de la exclusión. En Martín, M. y Sebastián, E (Ed.), Hacia un modelo educativo de calidad y transformador (pp. 177-184). Alcalá: Santillana.

Cabrerizo, J. y Rubio, M. (2007). Atención a la diversidad. Teoría y práctica. España: Prentice Hall.

CNCA. (2011). Estudio de caracterización de las Escuelas Artísticas. Recuperado de: https:// artistica.mineduc.cl/wp-content/uploads/sites/58/2016/04/ESTUDIO-ICNCA.pdf

. (2015). Estado de avance del Plan Nacional de Artes en la Educación 2015-2018. Santiago: CNCA.

Echeita, G. (2006). Educación para la inclusión o educación sin exclusiones. Madrid: Narcea.

Escarbajal, A., Mirete, A., Maquilón, J., Izquierdo, T., López, J., Orcajada, N. y Sánchez, M. (2012). La atención a la diversidad: la educación inclusiva. Revista Electrónica Interuniversitaria de Formación del Profesorado, 15(1), 135-144.

Escribano, A. y Martínez, A. (2013). Inclusión educativa y profesorado inclusivo. Aprender juntos para aprender a vivir juntos. Madrid: NARCEA.

Gairín, J. (1998). Estrategias organizativas en la atención a la diversidad. Educar, 22(23), 239-267. . (2012). La organización y atención a la diversidad en centros de educación secundaria de Iberoamérica. Reflexiones y experiencias. Santiago de Chile: Red Age.

García, F. y Delgado, M. (2017). Estrategias de enseñanza como respuesta a la diversidad: Concepciones y prácticas del pedagogo terapéutico. Revista Nacional e Internacional de Educación Inclusiva 10(1), 103-116.

García, X., Massani, J. y Bermúdez, I. (2016). La educación inclusiva en la formación de profesionales de la educación. Revista Científica de la Universidad de Cienfuegos, 8(1), 118-121.

Gómez, J. (2005). Pautas y estrategias para entender y atender a la diversidad en el aula. Pulso, (28), 199-214.

Graeme, F. (2003). Arte, educación y diversidad cultural. Barcelona: Paidós.

Hernández, R., Fernández, C. y Baptista, P. (2016). Metodología de la investigación. Sexta edición. México: McGraw-Hill/ Interamericana Editores.

Jiménez, C. (2011). La importancia de la Educación Artística en la formación integral del alumno. (Tesis de pregrado). Universidad Pedagógica Nacional, Cd. Del Carmen, México.

Martín, E. y Mauri, T. (1996). La atención a la diversidad en la educación secundaria. Barcelona: Horsori Editorial.

Moreno, A. (2016). La mediación artística: arte para la transformación social, la inclusión social y el desarrollo comunitario. Barcelona: Octaedro.

Organización de las Naciones Unidas para la Educación, la Ciencia y la Cultura (2009). Directrices sobre política de inclusión en la educación. París: UNESCO. 
Organización de las Naciones Unidas para la Educación, la Ciencia y la Cultura. (2017). Guía para asegurar la inclusión y la equidad en la educación. París: UNESCO.

Padilla, M. T. y Gil, J. (2008). La evaluación orientada al aprendizaje en la Educación Superior. Condiciones y estrategias para su aplicación en la docencia universitaria. Revista Española de Pedagogía, 66(241), 467-486.

Palacios, L. (2006). El valor del arte en el proceso educativo. Reencuentro, (46), 1-21.

Parra, C. (2011). Educación inclusiva: un modelo de diversidad humana. Revista Educación y Desarrollo Social, 5(1), 139-150.

Payà, A. (2017). Políticas de educación inclusiva en América Latina. Propuestas, realidades y retos de futuro. Revista Educación Inclusiva, 3(2), 125-142.

Rodríguez, D. y Gairín, J. (2015). Innovación, aprendizaje organizativo y gestión del conocimiento en las instituciones educativas. Educación, XXIV(46), 73-90.

Sanmartí, N. (2007). Diez ideas claves. Evaluar para aprender. Barcelona: Graó.

Stake, R. (2007). Investigación con estudio de caso. Madrid: Ediciones Morata.

Tomlison, C. (2005). Estrategias para trabajar con la diversidad en el aula. Buenos Aires: Paidós. 